\title{
Enhanced brightness in organic light-emitting diodes using a carbon nanotube composite as an electron-transport layer
}

\author{
P. Fournet, ${ }^{\text {a) }}$ J. N. Coleman, B. Lahr, A. Drury, and W. J. Blau \\ Department of Physics, Materials Ireland Polymer Research Center, Trinity College Dublin, \\ Dublin 2, Ireland \\ D. F. O'Brien \\ Nanomat Limited, Chemistry Building, University College Dublin, Belfield, Dublin 4, Ireland \\ H.-H. Hörhold \\ Institut für Organische und Makromolekulare Chemie, Friedrich-Schiller-Universität Jena, \\ Humboldtstraße 10, D-07743, Jena, Germany
}

(Received 26 February 2001; accepted for publication 8 May 2001)

\begin{abstract}
We have studied the effects of using a composite fabricated from carbon nanotubes and a host polymer, poly(m-phenylene-vinylene-co-2,5-dioctyloxy-p-phenylene-vinylene) (PmPV), as an electron-transport layer in organic light-emitting diodes. Double layer devices using this composite as an electron-transport layer, triple layer devices with a composite electron-transport layer, and poly(9-vinylcarbazole) as a hole-transport layer, as well as poly(2,5-dimethoxy-1,4phenylene-vinylene-2-methoxy-5(2'-ethylhexyloxy)-1,4-phenylene-vinylene (M3EH-PPV) single layer devices were prepared. Current-voltage-luminance and electroluminescent spectral measurements were performed using six different nanotube powder to polymer mass ratios $(0 \%$, $2 \%, 4 \%, 8 \%, 16 \%$, and $32 \%$ ) for all device structures studied. dc transport and photoluminescence behavior of the polymer-nanotube composite were also investigated. Although a potential barrier is introduced at the M3EH-PPV/composite interface, a significant increase in efficiency was observed using the composite. The best efficiency was obtained for those devices with an electron-transport layer of mass ratio $8 \%$. In addition, on doping with nanotubes, electron conductivity in the composite increased by over 4 orders of magnitude with little quenching of photoluminescence. (C) 2000 American Institute of Physics. [DOI: 10.1063/1.1383023]
\end{abstract}

\section{INTRODUCTION}

Advances in fabrication, ${ }^{1}$ purification, ${ }^{2-5}$ and processing techniques have allowed carbon nanotubes to show great potential in many areas of physics, chemistry, and engineering. Existing in two forms, multiwalled and single-walled, ${ }^{6}$ this form of carbon has been shown, both experimentally and theoretically, to have excellent mechanical and electrical properties. Individual nanotubes have elastic moduli ${ }^{7}$ in the region of $1 \mathrm{TPa}$, and in powder samples, bulk conductivity ${ }^{8}$ as high as $10^{5} \mathrm{~S} / \mathrm{m}$ has been measured.

However, while many potential applications have been suggested for carbon nanotubes, few practical uses have emerged. The main difficulties relate to the poor purity and processability of nanotube containing powders. Using nanotubes to improve the performance of existing functional organic devices would be one of the most accessible applications. One of the most studied of these device types over the last $10 \mathrm{yr}$ has been the organic light emitting diode (OLED).

Efficient organic electroluminescence $e^{9-11}$ requires the optimization of four factors: balance of injection of electrons and holes, transportation of these carriers as polarons within the polymer, recombination of carriers to form singlet excitons, and the radiative decay of these excitons. In order to

\footnotetext{
a) Author to whom correspondence should be addressed; electronic mail: fournetp@tcd.ie
}

achieve the optimization of these factors, heterojunctions using hole- and electron-transport layers are generally used.

While many organic materials can be used as holetransport layers, materials that work effectively as electrontransport layers are not so common. There are various reasons for this, $n$-type undoped polymers are rare while electron mobilities tend to be low as a result of the efficient trapping of negative carriers by impurities, etc. One possible alternative to polymers or molecular materials as electrontransport layers would be to use a high mobility material such as carbon nanotubes. In fact, these carbon nanocrystals can individually be extremely conductive with high mobilities ${ }^{6}$ for both electrons and holes.

Unfortunately, carbon nanotubes are not soluble in any known solvent and are too large to be deposited via thermal evaporation. In addition, using current production techniques, nanotube powders contain large quantities of graphitic impurities, which must be removed before the nanotubes can be used in any device application. However, recent work $^{4,5,12}$ shows that mixing nanotube powder with a solution of the conjugated polymer, poly (m-phenylene-vinyleneco-2,5-dioctyloxy-p-phenylene-vinylene) (PmPV), results in the selective interaction of PmPV strands and nanotubes. These polymer-coated nanotubes remain in solution while graphitic impurities sediment out. This impurity material can be removed by decantation, yielding a composite solution 
TABLE I. Chemicals, solvents, and film thickness for the different organic layers: ETL (electron-transport layer), EL (emissive layer), and HTL (hole-transport layer) used in this study.

\begin{tabular}{|c|c|c|c|c|}
\hline Layer & Material & Chemical structure & Solvent & $\begin{array}{c}\text { Thickness } \\
(\mathrm{nm})\end{array}$ \\
\hline ETL & $\begin{array}{l}\text { PmPV + } \\
\text { MWNT }\end{array}$ & + nanotubes & toluene & 110 \\
\hline EL & M3EH-PPV & & chlorobenzene & 110 \\
\hline HTL & PVK & & dichloromethane & 125 \\
\hline
\end{tabular}

rich in nanotubes, which can then be easily processed and spin coated into thin films.

In this article we study the use of a PmPV and a multiwalled carbon nanotube composite as an electron-transport layer in organic light-emitting diodes. A number of multilayer devices were fabricated incorporating electronand hole-transport layers, in conjunction with an emissive layer. The conjugated polymer, poly(2,5-dimethoxy1,4-phenylene-vinylene-2-methoxy-5(2'-ethylhexyloxy)-1,4phenylene-vinylene (M3EH-PPV) ${ }^{13}$ is used as the emissive layer. This polymer, which is a derivative of the well-known MEH-PPV, has been chosen because of its particularly good emissive properties. MEH-PPV with a small band gap of around $2.1 \mathrm{eV}$ has a low operating voltage for light emission and exhibits similar mobility properties for both holes and electrons. ${ }^{9,14,15}$ In order to stabilize the injection of holes, poly(9-vinylcarbazole) (PVK) was used. PVK represents a common hole-transport material because of its high hole mobility. ${ }^{9,16}$ Moreover, PVK with a band gap of $3.6 \mathrm{eV}$ is transparent to the photons emitted by the M3EH-PPV layer. Finally, the use of the polymer-nanotube composite with various nanotube powder to host polymer mass ratios for the electron-transport layer was studied.

\section{EXPERIMENTAL PROCEDURE}

The multiwalled carbon nanotubes used in this study were produced in a Krätschmer generator by the arc discharge method as described in previous works. ${ }^{17}$ The composite solutions were prepared by mixing the raw nanotube powder with PmPV in a toluene solution to obtain nanotube to polymer mass ratios of $0 \%, 2 \%, 4 \%, 8 \%, 16 \%$, and $32 \%$. It should be pointed out here that these values refer to nanotube powder to polymer mass ratios and do not reflect the subsequent removal of impurities by sedimentation and decantation. In order to disperse the nanotube powder the solution was ultrasonically agitated using a high power sonic tip for 1-2 min followed by $1-2 \mathrm{~h}$ in a low power sonic bath. After sonication the solutions were left to stand undisturbed for $48 \mathrm{~h}$ to allow any impurities present to sediment out. ${ }^{4,5}$ Finally the top $2 / 3$ of the solution were decanted giving solutions rich in nanotubes with virtually no impurities present. Homogenous composite films could then be prepared by simply spin coating the final solution. While solvent choice for the M3EH-PPV solution was motivated by the desire to avoid film mixing at the PVK/M3EH-PPV interface, this was not possible for the M3EH-PPV/composite interface. In fact, although chlorobenzene was used to dissolve M3EH-PPV, PmPV and M3EH-PPV share a common solvent, toluene and some mixing at this interface was therefore unavoidable when spin coating the composite film onto the M3EH-PPV layer. Work is ongoing to fabricate composites using a range of host materials with improved choice of solvents.

Shown in Table I are the molecular structures, solvents and thickness for the organic thin films used in this study. The OLED devices were fabricated by spin coating the different polymer solutions onto indium-tin-oxide (ITO) coated glass substrates and by thermally evaporating six aluminum (Al) electrodes onto the polymer layers. The overlap of the metal fingers and the ITO represents the active area (approximately $0.06 \mathrm{~cm}^{2}$ ).

The devices were tested in a vacuum chamber (using a rotary pump, pressure of $10^{-1} \mathrm{mbar}$ ). Contact to the external circuit was made via seven spring-loaded pins and silver paint.

The current-voltage characteristics were recorded using a Keithley 2400 sourcemeter, which was controlled by a Visual Basic program. The luminance characteristics were performed using this sourcemeter, a silicon photodiode, and a Keithley 2001 multimeter. The electroluminescence (EL) spectra were obtained using an Andor B401-UV charge coupled device (CCD) detector connected to an Oriel 77400 spectrograph. The light was collected by an Oriel 77863 optical fiber, which was placed directly in front of the devices. The CCD was controlled by a computer running an ANDOR 
custom software package. Photoluminescence measurements were carried out by exciting the samples with a Roithner NSHU590E narrow band UV diode and recorded as for EL measurements.

\section{RESULTS}

The devices studied in this work consisted of: (a) a single M3EH-PPV layer (to be referred to as SL: ITO/ $\mathrm{M} 3 \mathrm{EH}-\mathrm{PPV} / \mathrm{Al}$ ), (b) a double layered device consisting of PVK as a hole-transport layer and M3EH-PPV as the emissive layer (to be referred to as DLH: ITO/PVK/M3EH-PPV/ $\mathrm{Al}$ ), (c) six double layer devices with M3EH-PPV as the emissive layer and composite films of various mass ratios as the electron-transport layer (to be referred to as DLE: ITO/ M3EH-PPV/composite/Al), and (d) six triple layer devices with PVK as the hole-transport layer, M3EH-PPV as the emissive layer and composite films of various mass ratios as the electron-transport layer (to be referred to as TL: ITO/ $\mathrm{PVK} / \mathrm{M} 3 \mathrm{EH}-\mathrm{PPV} / \mathrm{composite/Al)}$. For all the samples listed above Current-voltage-luminescence characteristics and EL spectra were measured. In addition, in order to study the effects of the added nanotubes on the host polymer, single composite layers were studied for electron conductivity and photoluminescence quenching as a function of nanotube content. In the former case composite films of six different mass ratios $(0 \%, 2 \%, 4 \%, 6 \%, 8 \%, 16 \%$, and 32\%) were sandwiched between aluminum electrodes (Al/composite/Al) while in the latter case composite films were spin coated onto glass substrates.

Shown in Fig. 1 are typical current-voltage characteristics for the four types of OLEDs (SL, DLH, DLE and TL) used in this study. In the case of the DLE and TL devices the composite mass ratio was $8 \%$. It can clearly be seen that these curves tend to shift along the voltage axis from the SL to the TL device as device thickness increases. This can be characterized by an arbitrary voltage required to drive a current of $15 \mathrm{~mA} / \mathrm{cm}^{2}$ through each device. This "operating voltage" increases from 7.6 V in the SL to 13.2, 23.8, and $30.6 \mathrm{~V}$ in the DLH, DLE and TL devices, respectively.

Shown in Fig. 2 are luminance (in arbitrary units) versus current density plots for the four devices described in Fig. 1. These curves are linear in the low current density region as expected. These devices can be compared using the luminance at the arbitrary current density of $15 \mathrm{~mA} / \mathrm{cm}^{2}$. This "relative efficiency" value (in arbitrary units) increases from 3.4 for the SL device to 3.7, 15.8, and 19.0 for the DLH, DLE, and TL, respectively.

As mentioned previously, a range of nanotube mass ratios were used in the electron-transport layer for the DLE and TL devices. While only the results for the $8 \%$ composite devices are reported in Figs. 1 and 2, the operating voltage and relative efficiency for the whole range of electrontransport layers mass ratios are given in Table II. Two broad trends are observed. In the DLE, as the nanotube mass ratio is increased from $0 \%$ to $32 \%$ the operating voltage falls from 36.0 to 17.6 V. Similarly in the case of the TL device this voltage falls from 39.5 to $24.4 \mathrm{~V}$. However these voltages are still in excess of the operating voltages observed in the ab-

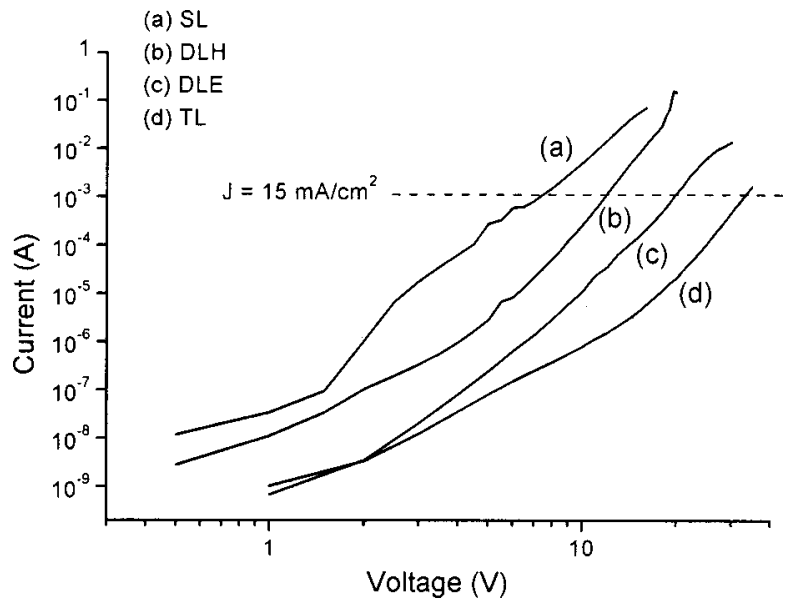

FIG. 1. Typical current-voltage characteristics for the main types of devices used in this work: SL (ITO/M3EH-PPV/Al), DLH (ITO/PVK/M3EH-PPV/ $\mathrm{Al}$ ), DLE (ITO/M3EH-PPV/composite/Al), and TL (ITO/PVK/M3EH-PPV/ composite/Al).

sence of the composite layer. These values were 7.6 and 13.2 $\mathrm{V}$ for the SL and DLH devices, respectively.

For the SL and DLH devices the relative efficiencies were 3.4 and 3.7 (in arbitrary units), respectively. For both the DLE and TL devices as the nanotube mass ratio increased, the relative efficiency rose to a maximum of 15.8 and 19.0, respectively, at a doping level of $8 \%$, before falling steadily to 2.9 and 5.3 at $32 \%$.

In addition, for the four devices described above EL spectra were measured and are presented in Fig. 3. For comparison purposes the EL spectrum for a single layer PmPV device is also shown. No significant spectral variations could be seen for any of the devices studied. For the four types of OLEDs used in this work, the spectra are consistent with emission from M3EH-PPV. No emission was observed from the low wavelength spectral region associated with EL from PmPV.

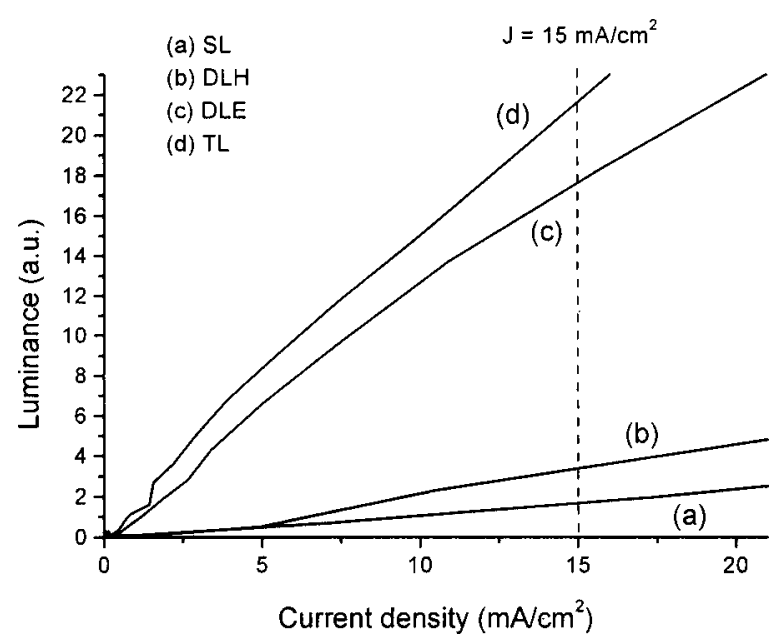

FIG. 2. Typical luminance-current relationships for the main types of devices: SL (ITO/M3EH-PPV/Al), DLH (ITO/PVK/M3EH-PPV/Al), DLE (ITO/M3EH-PPV/composite/Al), and TL (ITO/PVK/M3EH-PPV/ composite/Al). 
TABLE II. Operating voltage (arbitrary voltage required to drive 15 $\mathrm{mA} / \mathrm{cm}^{2}$ ) and relative efficiency (luminance at the arbitrary current density of $15 \mathrm{~mA} / \mathrm{cm}^{2}$ ) for each device: SL (ITO/M3EH-PPV/Al), DLH (ITO/PVK/ M3EH-PPV/Al), DLE (ITO/M3EH-PPV/composite/Al), and TL (ITO/PVK/ M3EH-PPV/composite/Al)

\begin{tabular}{ccccc}
\hline \hline Device & $\begin{array}{c}\text { Composite } \\
(\%)\end{array}$ & $\begin{array}{c}\text { Operating } \\
\text { voltage } \\
(\mathrm{V})\end{array}$ & $\begin{array}{c}\text { Effective } \\
\text { operating field } \\
(\text { V/nm) }\end{array}$ & $\begin{array}{c}\text { Relative } \\
\text { efficiency } \\
\text { (a.u. })\end{array}$ \\
\hline SL & & 7.6 & 0.076 & 3.4 \\
DLH & & 13.2 & 0.066 & 3.7 \\
DLE & 0 & 36.0 & 0.360 & 4.5 \\
& 2 & 26.7 & 0.267 & 6.5 \\
& 4 & 26.1 & 0.261 & 9.5 \\
& 8 & 23.8 & 0.238 & 15.8 \\
& 16 & 19.3 & 0.193 & 5.7 \\
TL & 32 & 17.6 & 0.176 & 2.9 \\
& 0 & 39.5 & 0.198 & 3.0 \\
& 2 & 32.4 & 0.162 & 8.8 \\
& 4 & 31.8 & 0.159 & 12.7 \\
& 8 & 30.6 & 0.153 & 19.0 \\
& 16 & 25.1 & 0.126 & 12.6 \\
& 32 & 24.4 & 0.122 & 5.3 \\
\hline \hline
\end{tabular}

In order to understand more completely the effect of embedding nanotubes in a polymer matrix, conductivity and photoluminescence measurements were carried out. The results of these are shown in Fig. 4. The conductivity of the host polymer used in this work, PmPV, was measured to be $1.32 \times 10^{-7} \mathrm{~S} / \mathrm{m}$. However, on addition of nanotubes the conductivity of the composite rapidly increased to 6.19 $\times 10^{-5} \mathrm{~S} / \mathrm{m}$ for the $2 \%$ sample. As the nanotube mass ratio was further increased the conductivity rose steadily to a plateau of $1.91 \times 10^{-3} \mathrm{~S} / \mathrm{m}$ for the $32 \%$ mass ratio sample. This represents an increase of approximately 5 orders of magnitude with respect to the host polymer. In the case of the photoluminescence the emission intensity decreased linearly with nanotube mass ratio. This decrease, however, was small compared with the increase in conductivity. In the case of the $32 \%$ sample the photoluminescence intensity had fallen by only $42 \%$ compared to the host polymer.

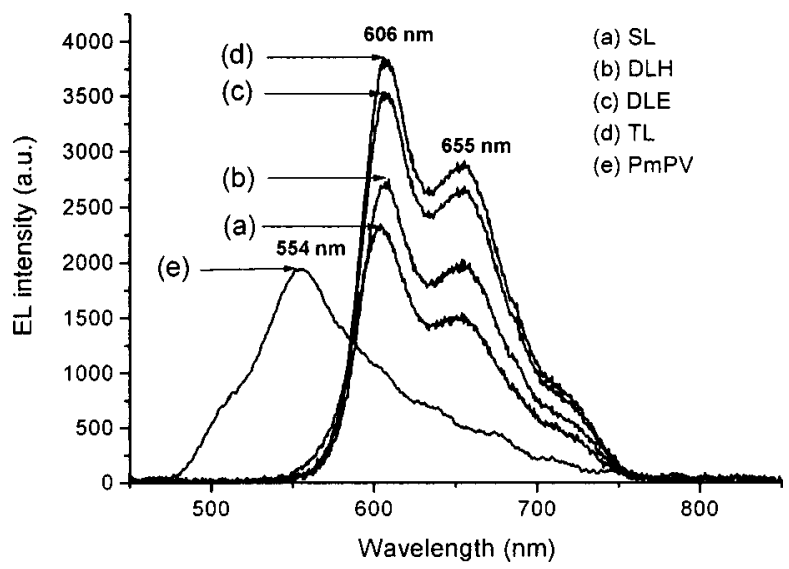

FIG. 3. Electroluminescence (EL) spectra for the SL, DLH, DLE, and TL devices. For comparison a PmPV single layer device EL spectrum is plotted. The spectra of the four different types of devices are consistent with emission from M3EH-PPV.

\section{DISCUSSION}

As shown in Fig. 1 the current-voltage curves for the four types of devices seem to show a clear trend with the largest current for a given voltage flowing through the SL device and the smallest current flowing in the TL device. However this graph is slightly misleading as these devices vary in thickness from approximately 110 to $345 \mathrm{~nm}$ for the SL and TL devices, respectively. Thus, the electric field is the more relevant parameter in this case. Consideration must also be given to the relative conductivities of the three individual layers. The conductivity of M3EH-PPV as estimated from Fig. 1 is approximately $5 \times 10^{-10} \mathrm{~S} / \mathrm{m}$, while PVK can be assumed to exhibit conductivity of similar magnitude. The conductivity of the $8 \%$ composite layer, however, is given in Fig. 4 as $2.83 \times 10^{-4} \mathrm{~S} / \mathrm{m}$. As this is 5 orders of magnitude higher than the other two materials we can assume that very little voltage is dropped across this layer. This suggests that virtually all of the applied voltage is dropped across the more resistive materials. Thus, we must use an effective thickness when calculating the effective field. This effective thickness is the combined thickness of the resistive layers in the device and does not include the thickness of the composite layer. Thus, the effective devices were 110, 235, 110, and $235 \mathrm{~nm}$ for the SL, DLH, DLE, and TL devices, respectively. Plots of current versus effective fields calculated using these thicknesses are presented in Fig. 5.

In this plot the four curves are clearly split into two groups of two. At lower field values are the SL and DLH current-effective field characteristics, while those for the DLE and TL devices are shifted up in field. The only difference between these groups is the presence of a composite electron-transport layer in the higher field curves. These data strongly suggest that for the materials used in this study the introduction of the electron-transport layer is accompanied by an increase in the potential barriers at the M3EH-PPV/ composite interface, blocking the passage of holes into the composite layer. Electron injection into the M3EH-PPV is probably also impeded at low nanotube doping levels. This is most likely due to the complicated energy structure in the composite with states associated with the semiconducting polymer and both semiconducting and metallic nanotubes.

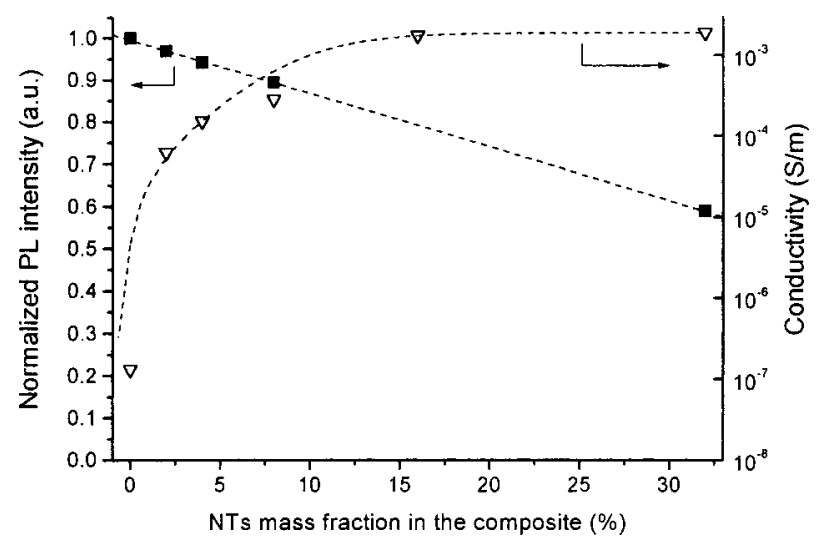

FIG. 4. Normalized photoluminescence (PL) intensity and conductivity for polymer and nanotube composite films as a function of nanotube to polymer mass ratio. 


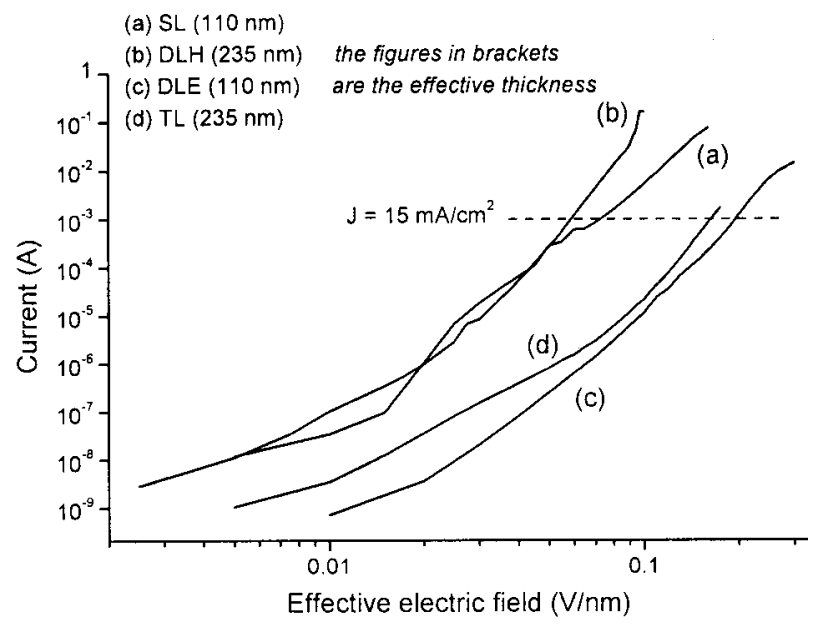

FIG. 5. Typical current-effective electric field characteristics for the main types of devices used in this work: SL, DLH, DLE, and TL. For each device the effective electric field represents the applied voltage divided by the effective thickness defined as the device thickness without considering the polymer-nanotube composite layer. The composite exhibits conductivity $10^{5}$ greater than PVK and M3EH-PPV so it could be assumed that almost no voltage would be dropped across the composite layer.

Shown in Fig. 6 is a proposed schematic diagram representing the band structure of a TL device. As PVK and M3EH-PPV are organic semiconductors, their Fermi energy levels lie close to the highest occupied molecular orbitals allowing efficient hole injection from the ITO anode. The situation is not so clear for the composite electron-transport layer. While aluminum has a work function of $4.2 \mathrm{eV}$, multiwalled carbon nanotubes have been experimentally shown to have a work function ${ }^{18}$ in the region of $4.8 \mathrm{eV}$. This should suggest that the composite should act as a hole-injection layer. However, EL is observed from the M3EH-PPV layer, so it is clear that electrons are injected from the cathode and through the composite layer. While it is probable that the difference between the nanotube work function and the M3EH-PPV lowest unoccupied molecular orbitals $(2.8 \mathrm{eV})$ is responsible for the potential barrier inferred from the currenteffective electric field data, this is probably not a complete picture. Field enhancement due to curvature effect at nanotube tips probably plays a more important role in the injection of electrons in the emissive layer.

The potential barrier however seems to be dependent on nanotube mass ratio. As the nanotube mass ratio is increased (not shown) the current flowing for a given voltage increases. This can be clearly seen in Fig. 7(b) and Table II where the operating voltage can be seen to fall as the mass ratio rises. This suggests that the potential barrier associated with the relative band energies of M3EH-PPV and the composite falls as more nanotubes are introduced into the system. This could possibly be explained by the formation of a new band as the nanotube concentration increases or curvature related field enhancement at the nanotube tips. However it should be noted that as the current in the DLE or TL devices never reaches the level observed in the SL, barrier effects are significant even at high nanotube mass ratios.

Another effect to be noted is that more current is observed in the TL device than in the DLE for a given voltage.

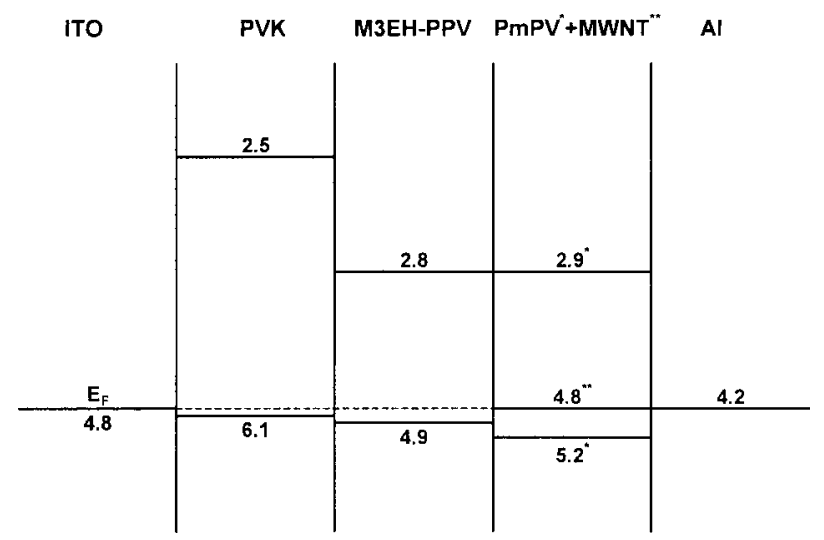

FIG. 6. Proposed schematic band diagram for a TL device (ITO/PVK/ M3EH-PPV/composite/Al) after equalization of Fermi energy levels. The HOMO and LUMO of the polymers (PVK, M3EH-PPV and PmPV) and work functions of electrodes (ITO, Al) and nanotubes are in $\mathrm{eV}$. The stars on $\mathrm{PmPV}$ and MWNT refer to the HOMO/LUMO of PmPV and work function of the nanotubes, respectively.

The difference between these structures is the presence of a hole-transport layer in the TL device. This result shows that this PVK layer acts to improve band matching and hence hole injection at the ITO electrode in these devices.

The EL spectra presented in Fig. 3 showed no spectral variations for all different devices with an emission wavelength characteristic of M3EH-PPV. Therefore, we can assume that every singlet exciton's radiative decay occurs in the M3EH-PPV layer. This result is of great importance since it shows that no radiative recombination occurs within the composite layer and emphasizes that the composite only acts to inject electrons into the emissive layer. Nevertheless these data do not exclude the formation of singlet excitons in the composite layer but show that their decays are nonradiative.

Shown in Fig. 7(a) is the EL at a given current as a function of nanotube mass ratio for the DLE and TL devices. This number is proportional to the relative efficiency of each device. For both DLE and TL devices, this efficiency value

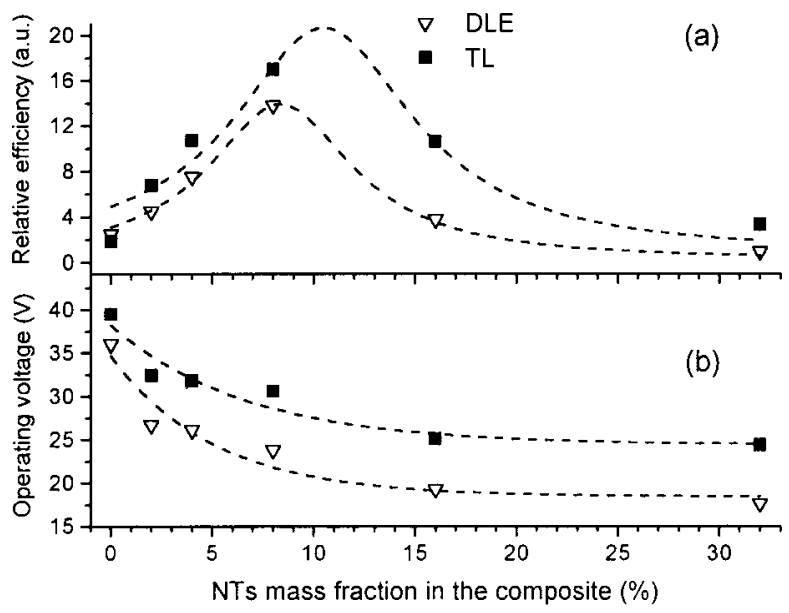

FIG. 7. (a) Relative efficiency (luminance at the arbitrary current density of $15 \mathrm{~mA} / \mathrm{cm}^{2}$ ), and (b) operating voltage (arbitrary voltage required to drive $15 \mathrm{~mA} / \mathrm{cm}^{2}$ ) as a function of nanotube to polymer mass ratio for the DLE (ITO/M3EH-PPV/composite/Al) and TL (ITO/PVK/M3EH-PPV/composite/ Al) devices. 
increases to a maximum at approximately $8 \%$ mass ratio before falling by $\sim 80 \%$ from the peak value. As both PVK and M3EH-PPV have good hole-transport properties it is probable that a significant number of holes travel from the ITO to the M3EH-PPV/composite interface where they are confined by the potential barrier caused by the difference in M3EH-PPV and composite band energies. Thus, it is probable that formation of singlet excitons is limited by the number of electrons entering the M3EH-PPV. Thus, as the nanotube mass ratio is increased and more electrons enter the M3EH-PPV, more excitons are formed and the luminescent intensity increases.

As electron injection into the M3EH-PPV increases with nanotube content, we would expect to see a plateau in the efficiency as the mass ratio is increased in the absence of any change in the hole current. However Fig. 7(a) shows that the efficiency reaches a maximum for the $8 \%$ sample before dropping to close to the $0 \%$ value by $32 \%$ mass ratio. This shows that this picture is too simplistic and some other effects must occur. While Fig. 4 shows that singlet excitons in PmPV formed by photoexcitation are quenched by the presence of nanotubes, this quenching is much less than expected. Photoluminescence (PL) studies on PPV doped $\mathrm{C}_{60}$ have shown that the PL is almost totally quenched at very low $\mathrm{C}_{60}$ concentrations. ${ }^{19}$ In the case of the PmPV nanotube composite studied in this work the PL intensity for the $32 \%$ sample was reduced by only $42 \%$ relative to the pure PmPV. Thus, even if all excitons were formed in the M3EH-PPV, near the interface, quenching due to nanotubes cannot explain a fall in EL of approximately $80 \%$ relative to the $8 \%$ doping composite TL device. Other quenching processes such as singlet-singlet exciton annihilation are also unlikely in this case.

While it is likely that the barrier to electrons at the $\mathrm{M} 3 \mathrm{EH}-\mathrm{PPV} / \mathrm{composite}$ interface falls as nanotube mass ratio increases, we have not considered changes in the barrier to holes traveling in the other direction. We have assumed that holes are confined in the M3EH-PPV by a potential barrier at the composite interface. Increased electron current then allows the formation of more excitons by better current balance. However if the barrier to holes was also dependent on nanotube mass ratio, this confinement could be reduced, resulting in the leakage of holes into the composite. As a result, even though we have more electrons in the recombination zone, we are losing holes faster due to leakage above $8 \%$ nanotube mass ratio. As a result less singlet excitons are formed resulting in lower EL intensity.

While the luminance efficiency (photons/injected charges) appears to increase in the presence of the composite electron-transport layer, current density flowing through the devices actually falls. This means that the device power efficiency (photons/Watt) is lowered upon the introduction of the carbon nanotubes. We have assumed that this is partly due to a barrier effect at the M3EH-PPV/composite interface. However, it must be noted that the composite layer was spin coated onto the M3EH-PPV layer. As toluene used for the composite is a common solvent for both materials, a degree of mixing can be expected at the interface. This is known to degrade carrier injection.
In fact, an increase in current injection of up to 6 orders of magnitude has been observed ${ }^{20}$ using the composite in heterojunctions with clean interfaces (Al/PMMA/composite/ $\mathrm{Al})$. This effect was not observed for devices where mixing had occurred (Al/PmPV/composite/Al). This suggests that electron injection from the composite could be substantially improved by careful choice, with regard to solvent, of the emissive layer or the use of host polymers, such as watersoluble polyvinylalcohol (PVA), in the composite material. Work is ongoing to characterize the use of carbon nanotubes in a PVA host as an electron-transport layer, as well as a hole-transport layer in M3EH-PPV based OLEDs.

\section{CONCLUSION}

In summary, we have investigated the use of a conjugated polymer-carbon nanotube composite as an electrontransport layer in M3EH-PPV based OLEDs. Introduction of the composite layer causes a potential barrier to both holes and electrons at the M3EH-PPV/composite interface. This barrier decreases with nanotube to polymer mass ratio leading to larger device currents. However, at all nanotube to polymer mass ratios used for the composite, the relative efficiencies for the devices using this material as an electrontransport layer have been improved compared to the M3EHPPV single layered devices. In addition, for all devices studied, the EL spectra showed exciton recombination to occur in the M3EH-PPV layer.

The optimum in the relative efficiency was observed for a nanotube mass ratio of $8 \%$. This suggests increased electron injection into the M3EH-PPV with increasing mass fraction coupled to increased hole leakage into the composite layer above $8 \%$.

This study has clearly demonstrated that carbon nanotubes can be used as a functional material in organic devices. Research characterizing the polymer and carbon nanotube composite has determined that the nanotubes can be easily purified, processed and then used in functional all organic devices. OLEDs have been successfully tested and we can envisage that nanotubes could be used, in such a form, in organic solar cells, transistors, or field emission displays.

\section{ACKNOWLEDGMENTS}

The authors wish to acknowledge the Irish High Education Authority (HEA), as well as the European Union (EU) for their support of this work.

${ }^{1}$ C. Journet, W. K. Maser, P. Bernier, A. Loiseau, M. L. de la Chapelle, S. Lefrant, P. Deniard, R. Lee, and J. E. Fischer, Nature (London) 388, 756 (1997).

${ }^{2}$ P. M. Ajayan and T. W. Ebbesen, Rep. Prog. Phys. 60, 1025 (1997).

${ }^{3}$ G. S. Duesberg, W. J. Blau, H. J. Byrne, J. Muster, M. Burghard, and S. Roth, Synth. Met. 103, 2484 (1999).

${ }^{4}$ J. N. Coleman, D. F. O'Brien, A. B. Dalton, B. McCarthy, B. Lahr, R. C. Barklie, and W. J. Blau, J. Chem. Phys. 113, 9788 (2000).

${ }^{5}$ J. N. Coleman, D. F. O'Brien, A. B. Dalton, B. McCarthy, B. Lahr, A. Drury, R. C. Barklie, and W. J. Blau, Chem. Commun. (Cambridge) 20, 2000 (2000)

${ }^{6}$ M. S. Dresselhaus, G. Dresselhaus, P. C. Eklund, Science of Fullerenes and Carbon Nanotubes (Academic, New York, 1995).

${ }^{7}$ M. Tracey, T. Ebbensen, and J. Gibson, Nature (London) 381, 678 (1990).

${ }^{8}$ A. B. Kaiser, G. S. Duesberg, and S. Roth, Phys. Rev. B 57, 1418 (1998). 
${ }^{9}$ M. Pope and C. E. Swenberg, Electronic Process in Organic Crystals and Polymers (Oxford University Press, New York, 1999).

${ }^{10}$ R. H. Friend et al., Nature (London) 397, 121 (1999).

${ }^{11}$ J. Kalinowski, J. Phys. D 32, R179 (1999).

${ }^{12}$ A. B. Dalton, C. Stephan, J. N. Coleman, B. McCarthy, P. M. Ajayan, S. Lefrant, P. Bernier, W. J. Blau, and H. J. Byrne, J. Phys. Chem. B 104, 10012 (2000).

${ }^{13}$ S. Pfeiffer and H.-H. Hörhold, Macromol. Chem. Phys. 200, 1870 (1999).

${ }^{14}$ J. Campbell Scott, P. J. Brock, J. R. Salem, S. Ramos, G. G. Malliaras, S.

A. Carter, and L. Bozano, Synth. Met. 111-112, 289 (2000).
${ }^{15}$ I. D. Parker, J. Appl. Phys. 75, 1656 (1994).

${ }^{16}$ Y. Yang, Q. Pei, and A. J. Heeger, J. Appl. Phys. 79, 934 (1996).

${ }^{17}$ W. Krätschmer, L. D. Lamb, K. Fostiropoulos, and D. R. Huffman, Nature (London) 347, 354 (1990).

${ }^{18}$ H. Ago, Th. Kugler, F. Cacialli, R. H. Friend, W. R. Salaneck, Y. Ono, T. Yamabe, and K. Tanaka, Synth. Met. 103, 2494 (1999).

${ }^{19}$ N. S. Sariciftci, L. Smilowitz, A. J. Heeger, and F. Wudl, Science 258, 1474 (1992)

${ }^{20}$ B. E. Kilbride, J. N. Coleman, D. F. O'Brien, and W. J. Blau, Synth. Met. 121, 1227 (2001). 